Research Article

\title{
Introducing New Exponential Zagreb Indices for Graphs
}

\author{
Nihat Akgunes $(\mathbb{D}$ and Busra Aydin \\ Department of Mathematics-Computer Sciences, Necmettin Erbakan University, Konya 42090, Turkey \\ Correspondence should be addressed to Nihat Akgunes; nakgunes@erbakan.edu.tr
}

Received 12 December 2020; Accepted 10 May 2021; Published 26 May 2021

Academic Editor: Efthymios G. Tsionas

Copyright (C) 2021 Nihat Akgunes and Busra Aydin. This is an open access article distributed under the Creative Commons Attribution License, which permits unrestricted use, distribution, and reproduction in any medium, provided the original work is properly cited.

New graph invariants, named exponential Zagreb indices, are introduced for more than one type of Zagreb index. After that, in terms of exponential Zagreb indices, lists on equality results over special graphs are presented as well as some new bounds on unicyclic, acyclic, and general graphs are obtained. Moreover, these new graph invariants are determined for some graph operations.

\section{Introduction and Preliminaries}

Let $G$ be a simple and connected graph with vertex set $V(G)=\left\{v_{1}, v_{2}, \ldots, v_{n}\right\}$ and edge set $E(G)$ such that $|E(G)|=m$. If any two vertices $u, v \in V(G)$ are adjacent with an edge, they are denoted by $u v \in E(G)$. The degree of any vertex $v \in V(G)$ is the number of edges that are incident to $v$ and denoted by $\operatorname{deg}_{G}(v)$. In this paper, we will generally follow reference [1] for unexplained terminology and notation.

In chemical graph theory, different chemical structures are usually modeled by a molecular graph to understand different properties of the chemical compound theoretically. A graph invariant that correlates the physico-chemical properties of a molecular graph with a number is called a molecular structure index. By use of the adjacency, degree, or distance matrices in graph theory, one can describe the structure of molecules in chemistry. The application of molecular structure indices is a standard procedure in structure-property relations, i.e., in QSPR/QSAR studies [2]. As studied in [3], some chemically interesting graphs can be obtained by different graph operations applied onto some general or particular graphs, so it is essential to study such graph operations to understand how they are related to the corresponding topological indices of the original graphs. Some of topological indices are vertex-degree-based, the others are distance or eccentricity-based.
The first vertex-degree-based index, called Zagreb, was introduced in $[4,5]$ under the name of first and second Zagreb indices $M_{1}(G)=\sum_{v \in V(G)} \operatorname{deg}(v)^{2} \quad$ and $M_{2}(G)=\sum_{u v \in E(G)} \operatorname{deg}(u) \operatorname{deg}(v)$, respectively. The first Zagreb index can also be defined as $M_{1}(G)=\sum_{u v \in E(G)}[\operatorname{deg}(u)+\operatorname{deg}(v)]$ which is used to modeling the best structure-boiling point of alkanes (cf. [6]). Zagreb indices are the oldest and extremely studied graph invariant among others. Readers can refer to the papers, for instance, [6-15] and citations therein. Although there are so many different types of Zagreb indices other than first and second Zagreb indices introduced in the literature, the most common and taken attentions are the first and second multiplicative Zagreb and multiplicative-sum Zagreb indices (see, for instance, [16-19]), Zagreb co-indices (see, for instance, [20]), and finally the multiplicative Zagreb coindices (see, for instance, $[8,9,20-23]$ ), and citations therein.

Later on, Rada [24] has recently introduced a function of exponential topological indices as $e^{\varphi}$ for a vertex-degreebased index $\varphi$ and also has studied Randic's exponential index over the set of graphs with $n$ nonisolated vertices. Furthermore, by using the same approximation $e^{\varphi}$, Cruz and Rada [25] have investigated extremal tree graphs for exponential first and second Zagreb indices except maximal tree graph of exponential second Zagreb index. More recently, in [26], the maximal tree graph for exponential second Zagreb index has been obtained. In all these 
references, the authors defined exponential vertex-degreebased topological indices as the power of exponential.

In the light of the thoughts in [24-26], we will first introduce various types of exponential Zagreb indices with a different manner rather than the references in this paragraph. After that, we will investigate some properties over them in separate sections.

Definition 1. For the graph $G$,

(i) The first and second exponential Zagreb indices are defined by

$$
\mathrm{EM}_{1}(G)=\sum_{v \in V(G)} e^{\operatorname{deg}(v)^{2}} \text { and } \operatorname{EM}_{2}(G)=\sum_{u v \in E(G)} e^{\operatorname{deg}(u) \operatorname{deg}(v)} .
$$

(ii) The sum version of the first exponential Zagreb index is given by

$$
E M_{1}^{*}(G)=\sum_{u v \in E(G)} e^{\operatorname{deg}(u)+\operatorname{deg}(v)}
$$

(iii) The multiplicative exponential Zagreb indices are defined by

$$
E \Pi_{1}(G)=\prod_{v \in V(G)} e^{\operatorname{deg}(v)^{2}} \text { and } E \Pi_{2}(G)=\prod_{u v \in E(G)} e^{\operatorname{deg}(u) \operatorname{deg}(v)},
$$

respectively.

By considering exponential Zagreb (co)indices given in Definition 1 and investigating some new theories via these indices, we aim to point out a new studying field of Zagreb indices for a possible usage in mathematics and mathematical chemistry.

The organization of this paper is as follows. We will first present the equalities of the various exponential Zagreb indices obtained by the number of degrees and the number of vertices over well-known graphs. After that, we will show some results on the comparison between exponential Zagreb indices and bounds. Furthermore, we will prove the existence of some new bounds via these new indices over unicyclic and acyclic graphs. Finally, we will state and prove some theorems on graph operations for the new exponential Zagreb indices.

\section{Results on Special Graphs}

In this section, we will present some theorems without proofs since they can be obtained easily by considering the index formulas indicated in Definition 1. In detail, we will determine all exponential Zagreb indices for cycle $C_{n}$, complete $K_{n}$, path $P_{n}$, star $S_{n}$, wheel $W_{n}$, and complete bipartite graphs $K_{m, n}$, respectively.

A direct calculation, by considering the first part of (1) and (2), implies the following theorem.
Theorem 1. The list on the first exponential Zagreb index and the sum version of the first exponential Zagreb index are

$$
\operatorname{EM}_{1}(G)= \begin{cases}n \cdot e^{4} & \text { if } G=C_{n}, \text { for } n \geq 3, \\ n \cdot e^{(n-1)^{2}} & \text { if } G=K_{n}, \text { for } n \geq 3, \\ 2 e+(n-2) \cdot e^{4} & \text { if } G=P_{n}, \text { for } n \geq 2, \\ (n-1) \cdot e+e^{(n-1)^{2}} & \text { if } G=S_{n}, \text { for } n \geq 2, \\ (n-1) \cdot e^{9}+e^{(n-1)^{2}} & \text { if } G=W_{n}, \text { for } n \geq 4, \\ m \cdot e^{n^{2}}+n \cdot e^{m^{2}} & \text { if } G=K_{m, n}, \text { for } m, n \geq 1,\end{cases}
$$

$$
E M_{1}^{*}(G)= \begin{cases}n \cdot e^{4} & \text { if } G=C_{n}, \text { for } n \geq 3, \\ \frac{1}{2}(n-1) \cdot n \cdot e^{2(n-1)} & \text { if } G=K_{n}, \text { for } n \geq 3, \\ 2 e^{3}+(n-3) \cdot e^{4} & \text { if } G=P_{n}, \text { for } n \geq 2, \\ (n-1) e^{n} & \text { if } G=S_{n}, \text { for } n \geq 2, \\ (n-1) \cdot e^{6}+(n-1) e^{n+2} & \text { if } G=W_{n}, \text { for } n \geq 4, \\ m \cdot n \cdot e^{m+n} & \text { if } G=K_{m, n}, \text { for } m, n \geq 1,\end{cases}
$$

respectively.

By the second part of (1), we obtain the second exponential Zagreb index for the same graphs as in the next theorem.

\section{Theorem 2.}

$E M_{2}(G)= \begin{cases}n \cdot e^{4} & \text { if } G=C_{n}, n \geq 3, \\ \frac{1}{2} \cdot(n-1) n \cdot e^{(n-1)^{2}} & \text { if } G=K_{n}, \text { for } n \geq 3, \\ 2 e^{2}+(n-3) \cdot e^{4} & \text { if } G=P_{n}, \text { for } n \geq 2, \\ (n-1) \cdot e^{(n-1)} & \text { if } G=S_{n}, \text { for } n \geq 2, \\ (n-1) \cdot e^{9}+(n-1) e^{3(n-1)} & \text { if } G=W_{n}, \text { for } n \geq 4, \\ m n \cdot e^{m n} & \text { if } G=K_{m, n}, \text { for } m, n \geq 1 .\end{cases}$

Theorem 3. By (3), lists on the multiplicative exponential Zagreb indices for some special graphs are as follows: 


$$
\begin{aligned}
& E \Pi_{1}(G)= \begin{cases}e^{4 n} & \text { if } G=C_{n}, \text { for } n \geq 3, \\
e^{n(n-1)^{2}} & \text { if } G=K_{n}, \text { for } n \geq 3, \\
e^{4 n-6} & \text { if } G=P_{n}, \text { for } n \geq 2, \\
e^{n(n-1)} & \text { if } G=S_{n}, \text { for } n \geq 2, \\
e^{(n-1)(n+8)} & \text { if } G=W_{n}, \text { for } n \geq 4, \\
e^{m n(m+n)} & \text { if } G=K_{m, n}, \text { for } m, n \geq 1,\end{cases} \\
& E \Pi_{2}(G)= \begin{cases}e^{4 n} & \text { if } G=C_{n}, \text { for } n \geq 3, \\
e^{\frac{1}{2} n(n-1)^{3}} & \text { if } G=K_{n}, \text { for } n \geq 3, \\
e^{4 n-8} & \text { if } G=P_{n}, \text { for } n \geq 2, \\
e^{(n-1)^{2}} & \text { if } G=S_{n}, \text { for } n \geq 2, \\
e^{3(n-1)(n+2)} & \text { if } G=W_{n}, \text { for } n \geq 4, \\
e^{(m n)^{2}} & \text { if } G=K_{m, n}, \text { for } m, n \geq 1 .\end{cases}
\end{aligned}
$$

Theorem 4. By the first part of (7), lists on the additive exponential Zagreb co-indices are as follows:

$$
\overline{E M_{1}}(G)= \begin{cases}\frac{(n-3) n}{2} e^{4} & \text { if } G=C_{n}, \text { for } n \geq 3, \\ 0 & \text { if } G=K_{n}, \text { for } n \geq 3, \\ e^{2}+2(n-3) e^{3}+\frac{(n-4)(n-3)}{2} e^{4} & \text { if } G=P_{n}, \text { for } n \geq 2, \\ \frac{(n-2)(n-1)}{2} e^{2} & \text { if } G=S_{n}, \text { for } n \geq 2, \\ \frac{(n-4)(n-1)}{2} e^{6} & \text { if } G=W_{n}, \text { for } n \geq 4, \\ \frac{(m-1) m}{2} e^{2 n}+\frac{(n-1) n}{2} e^{2 m} & \text { if } G=K_{m, n}, \text { for } m, n \geq 1,\end{cases}
$$




$$
\overline{E M_{2}}(G)= \begin{cases}\frac{(n-3) n}{2} e^{4} & \text { if } G=C_{n}, \text { for } n \geq 3, \\ 0 & \text { if } G=K_{n}, \text { for } n \geq 3, \\ e+2(n-3) e^{3}+\frac{(n-4)(n-3)}{2} e^{4} & \text { if } G=P_{n}, \text { for } n \geq 2, \\ \frac{(n-2)(n-1)}{2} e & \text { if } G=S_{n}, \text { for } n \geq 2, \\ \frac{(n-4)(n-1)}{2} e^{9} & \text { if } G=W_{n}, \text { for } n \geq 4, \\ \frac{(m-1) m}{2} e^{n^{2}}+\frac{(n-1) n}{2} e^{m^{2}} & \text { if } G=K_{m, n}, \text { for } m, n \geq 1 .\end{cases}
$$

Theorem 5. By the second part of (7), lists on the multiplicative exponential Zagreb coindices are as follows:

$\overline{E \Pi_{1}}(G)= \begin{cases}e^{2(n-3) n} & \text { if } G=C_{n}, \text { for } n \geq 3, \\ 0 & \text { if } G=K_{n}, \text { for } n \geq 3, \\ e^{6 n+2(n-3)(n-4)-16} & \text { if } G=P_{n}, \text { for } n \geq 2, \\ e^{(n-1)(n-2)} & \text { if } G=S_{n}, \text { for } n \geq 2, \\ e^{3(n-1)(n-4)} & \text { if } G=W_{n}, \text { for } n \geq 4, \\ e^{(m+n-2) m n} & \text { if } G=K_{m, n}, \text { for } m, n \geq 1,\end{cases}$

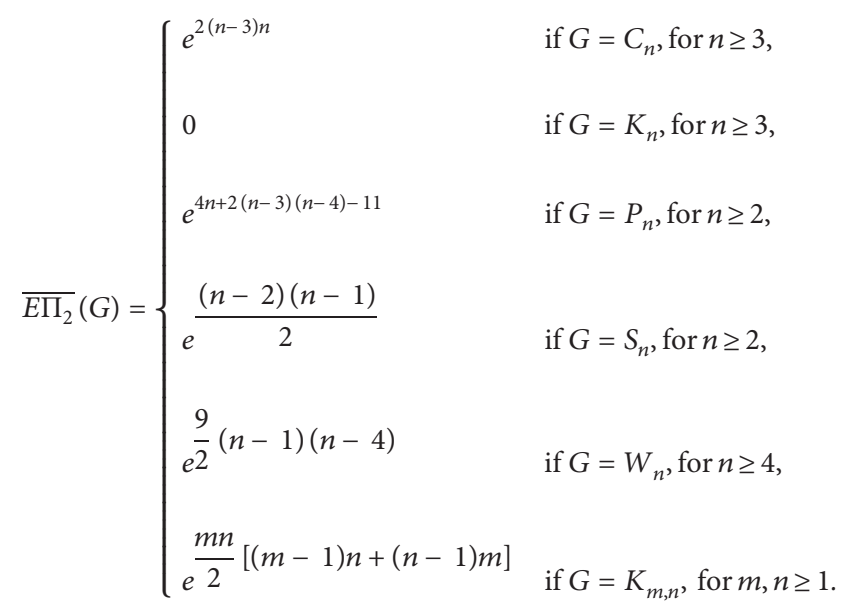

\section{Results on the Comparison between Exponential Zagreb Indices and Some Bounds}

The goal of this section is to give some results on the comparison between exponential Zagreb indices and bounds. We need to remind the following facts for our results:

Fact 1: it is quite clear that exponential Zagreb index is greater than an ordinary Zagreb index since $e^{i}>i$ for $\forall i>0$

Fact 2: for any list of $n$ nonnegative real numbers $x_{1}, x_{2}, \ldots, x_{n}$, it is well known that the inequality of arithmetic and geometric means (AM-GM inequality) is defined by $x_{1}+x_{2}+\cdots+x_{n} / n \geq \sqrt{[n]} x_{1} x_{2} \ldots x_{n}$ and the equality holds if and only if $x_{1}=x_{2}=\cdots=x_{n}$

Theorem 6. For a graph $G$ with $n$ vertices and $m$ edges, we have $E \Pi_{1}(G) \geq e^{4 m^{2} / n}$.

Proof. By (3) and Cauchy-Schwarz inequality, we obtain

$$
\begin{aligned}
E \Pi_{1}(G) & =\prod_{u \in V(G)} e^{\operatorname{deg}(u)^{2}}=e^{d_{1}^{2}+d_{2}^{2}+\cdots+d_{n}^{2}} \\
& =e^{1 / n \cdot\left(d_{1}^{2}+d_{2}^{2}+\cdots+d_{n}^{2}\right) \cdot\left(1^{2}+1^{2}+\cdots+1^{2}\right)} \geq e^{1 / n \cdot\left(d_{1}+d_{2}+\cdots+d_{n}\right)^{2}} \\
& =e^{1 / n \cdot(2 m)^{2}}=e^{4 m^{2} / n}
\end{aligned}
$$

as required. 
Lemma 1 (see [27]). For any graph $G$ and any edge $u v \in E(G)$,

$$
\prod_{u v \in E(G)} \operatorname{deg}(u) \operatorname{deg}(v)=\prod_{u \in V(G)} \operatorname{deg}(u)^{\operatorname{deg}(u)} .
$$

Theorem 7. For a graph $G$ with $n$ vertices and $m$ edges, $E \Pi_{2}(G) \geq e^{(2 m / n)^{2 m}}$.

Proof. By equation (3), Fact 2, and Lemma 1, we have

$$
E \Pi_{2}(G)=e^{\sum_{u v E(G)} \operatorname{deg}(u) \operatorname{deg}(v)}=e^{m} \cdot \sum_{u v \in E(G)} \frac{\operatorname{deg}(u) \operatorname{deg}(v)}{m} \geq e^{m} \cdot \sqrt[m]{\prod_{u v \in E(G)} \operatorname{deg}(u) \operatorname{deg}(v)}=e^{m} \cdot \sqrt[m]{\prod_{u \in V(G)} \operatorname{deg}(u)^{\operatorname{deg}(u)}} .
$$
obtain

By applying ln into the AM-GM inequality above, we

$$
\begin{aligned}
e^{m} \cdot \ln \sum_{u v \in E(G)} \frac{\operatorname{deg}(u) \operatorname{deg}(v)}{m} & \geq e^{m} \cdot \ln \sqrt[m]{\prod_{u \in V(G)} \operatorname{deg}(u)^{\operatorname{deg}(u)}} \\
& =e^{m} \cdot \frac{1}{m} \cdot \sum_{i=1}^{n} d_{i} \cdot \ln d_{i}
\end{aligned}
$$

(where each $d_{i}$ is the degree of the vertex $u \in V(G)$ )

$$
\geq e^{\sum_{i=1}^{n} d_{i} \cdot \ln \sum_{i=1}^{n} d_{i} / n}=e^{\sum_{i=1}^{n} d_{i} \cdot \ln 2 m / n}=e^{2 m \cdot \ln 2 m / n}=\left(\frac{2 m}{n}\right)^{2 m},
$$

which implies

$$
\left(\sum_{u v \in E(G)} \frac{\operatorname{deg}(u) \operatorname{deg}(v)}{m}\right)^{m} \geq\left(\frac{2 m}{n}\right)^{2 m} \longrightarrow e^{\frac{1}{m}} \sum_{u v \in E(G)} \operatorname{deg}(u) \operatorname{deg}(v) \geq e^{\left(\frac{2 m}{n}\right)^{2}}
$$

Henceforth,

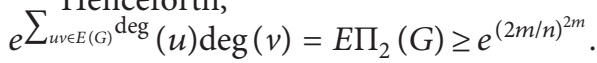

We have the following result between two multiplicative exponential Zagreb indices.

Theorem 8. For a simple, connected graph $G$ with $n$ vertices and $m$ edges, let $d_{i}$ be the degree of any vertex $v_{i} \in V(G)$ and $\Delta$ be the maximum degree of $G$. Then, $\left(E \Pi_{2}(G)\right)^{2} \leq\left(E \Pi_{1}(G)\right)^{\Delta}$.

Proof. Recall that the first and second Zagreb indices are defined as (Textranslation failed) and (Tex translation failed), respectively. Now, for $i, j \in\{1,2, \ldots, n\}$ and $j \leq i$, by the fact $d_{i}^{2}+d_{j}^{2} \leq d_{i} \cdot d_{i}^{2}$, we obtain

$$
\begin{aligned}
\left(E \Pi_{2}(G)\right)^{2} & =e^{2 \cdot M_{2}(G)}=e^{2 \sum d_{i} d_{j}}=e^{\sum 2 d_{i} d_{j}} \leq e^{\sum\left(d_{i}^{2}+d_{j}^{2}\right)} \\
& \leq e^{\sum d_{i} \cdot d_{i}^{2}} \leq e^{\sum \Delta \cdot d_{i}^{2}}=e^{\Delta \cdot M_{1}(G)}=\left(E \Pi_{1}(G)\right)^{\Delta},
\end{aligned}
$$

where each summation defined under the condition $i \sim j$. Hence, the result is obtained.

A relation between second exponential Zagreb index and second multiplicative exponential Zagreb index can be given in the next result.

Theorem 9. For any graph $G$ with $m$ edges, it is always true that $\operatorname{EM}_{2}(G) / m \geq \sqrt[m]{E \Pi_{2}(G)}$.

Proof. Since $\mathrm{EM}_{2}(G)$ in equation (1) is defined as (Tex translation failed), by considering Fact 2, we have 


$$
\frac{\operatorname{EM}_{2}(G)}{m}=\frac{\sum_{i \sim j} e^{d_{i} d_{j}}}{m} \geq \sqrt[m]{\prod_{i \sim j} e^{d_{i} d_{j}}=\sqrt[m]{E \Pi_{2}(G)}}
$$

which gives the result.

There exist the following lower and upper bounds for the sum version of the first exponential Zagreb index $\operatorname{EM}_{1}^{*}(G)$ defined in equation (2).

Theorem 10. For a graph $G$, we have n.e $e^{4} \leq M_{1}^{*}(G) \leq$ $(n-1) n / 2 e^{2(n-1)}$. Moreover, the equality for lower bound holds if $G \simeq C_{n}$ and the equality holds for the upper bound holds if $G \simeq K_{n}$.

Proof. As usual, let $d_{v}$ be the degree of any vertex $v \in V(G)$. It is clear that equation (2) can be notated as (Tex translation failed). Since we are interested in the degrees of adjacent vertices in $E M_{1}^{*}(G)$, it is obvious that $K_{n}$ has the largest degree of all adjacent vertices. Then, we obtain

$$
E M_{1}^{*}\left(K_{n}\right)=\sum_{u \sim u} e^{(n-1)+(n-1)}=\frac{(n-1) n}{2} e^{2(n-1)},
$$

which implies that $K_{n}$ is the upper bound for $\operatorname{EM}_{1}^{*}(G)$.

Recall that the multiplicative-sum Zagreb index [17] is defined by (Tex translation failed), and according to [16], the lower bound for it is either $C_{n}$ or $P_{n}$. Now, by giving attention to the construction of $E M_{1}^{*}(G)$, we easily see that $C_{n}$ or $P_{n}$ will be a lower bound. However, $E M_{1}^{*}\left(P_{n}\right)=2 e^{3}+$ $(n-3) e^{4}>n \cdot e^{4}=E M_{1}^{*}\left(C_{n}\right)$ implies that $C_{n}$ is the lowest bound for $E M_{1}^{*}(G)$.

As we mentioned in Section 1, following up [24], Cruz and Rada (Theorem 2.7in [25]) studied about the extremal tree graphs for the exponential first and second Zagreb indices. The result did not cover the maximal tree graph for the exponential of the second Zagreb index. Recently, in Lemma 3 in [26], Zeng and Deng obtained this gap. We should remind that the definition of exponential Zagreb indices in these papers is a different approximation than our way presented in Definition 1.

In the following, we will present some results related to the exponential Zagreb and multiplicative exponential Zagreb indices on any simple graphs as well as extremal tree graphs. In fact, proofs of the remaining theorems in this section will be based on the lemmas that will be stated just before results and also based on the truth Fact 1 which was stated at the beginning of this section.

Lemma 2 (see [28]). Among n-vertex tree graphs, the star graph has maximum and the path graph has minimum value of the first Zagreb index. In other words, $M_{1}\left(P_{n}\right) \leq M_{1}\left(T_{n}\right) \leq M_{1}\left(S_{n}\right)$.

Theorem 11. For any tree graph $T_{n}$ with $n$-vertices, we certainly have $E M_{1}\left(P_{n}\right) \leq E M_{1}\left(T_{n}\right) \leq E M_{1}\left(S_{n}\right)$ and $E \Pi_{1}\left(P_{n}\right) \leq E \Pi_{1}\left(T_{n}\right) \leq E \Pi_{1}\left(S_{n}\right)$.
The proof of the above theorem can be seen directly by adapting the first parts of equations in (1) and (3) into Lemma 2.

Theorem 12. For any graph $G$ with $n$-vertices, there exist the orderings $E M_{1}(G) \leq E M_{1}\left(K_{n}\right)$ and $E \Pi_{1}(G) \leq E \Pi_{1}\left(K_{n}\right)$.

Proof. It is well known that the maximum degree of any vertex in a simple and connected graph with $n$ vertices is $n-1$. Thus, (Tex translation failed).

On the contrary, the product $\prod$ clearly provides the above inequality, and hence, the result is obtained.

In [29], Das and Gutman showed that the second Zagreb index $M_{2}(G)$ decreases when the edges delete from a graph. For this reason, graphs having the greatest and smallest number of edges have maximum and minimum $M_{2}(G)$, respectively. With this idea, clearly, the graph with maximum value of second Zagreb index is actually the complete graph as depicted in the next lemma.

Lemma 3 (see [29]). If $G$ has $n$ vertices, then $M_{2}\left(\overline{K_{n}}\right) \leq M_{2}(G) \leq M_{2}\left(K_{n}\right)$.

Theorem 13. For any graph $G$ with $n$ vertices, the maximum second exponential Zagreb and the maximum multiplicative second exponential Zagreb indices have orderings:

$$
E M_{2}(G) \leq E M_{2}\left(K_{n}\right) \text { and } E \Pi_{2}(G) \leq E \Pi_{2}\left(K_{n}\right),
$$

respectively.

Proof. Since the second parts of equations (1) and (3) can also be notated as $E_{2}(G)=\sum_{u v \in E(G)} e^{M_{2}(G)}$ and $E \Pi_{2}(G)=\prod_{u v \in E(G)} e^{M_{2}(G)}, \quad$ respectively, and since $e^{M_{2}(G)} \leq e^{M_{2}\left(K_{n}\right)}$ holds by Lemma 3 , we reach the required orderings. follows.

The second Zagreb index version of Lemma 2 is as

Lemma 4 (see [29]). Among n-vertex tree graphs, the star graph has the maximum value and the path graph has the minimum value of the second Zagreb index. In other words, $M_{2}\left(P_{n}\right) \leq M_{2}\left(T_{n}\right) \leq M_{2}\left(S_{n}\right)$.

Now, by adapting the second parts of equations (1) and (3) in Lemma 4, the following result follows immediately.

Theorem 14. For any tree graph $T_{n}$ with $n$-vertices, we definitely have $E M_{2}\left(P_{n}\right) \leq E M_{2}\left(T_{n}\right) \leq E M_{2}\left(S_{n}\right)$ and $E \Pi_{2}\left(P_{n}\right) \leq E \Pi_{2}\left(T_{n}\right) \leq E \Pi_{2}\left(S_{n}\right)$.

\section{Results on Unicyclic and Acyclic Graphs}

It is known that topological indices are often used for molecular graphs, and unicyclic, bicyclic, and acyclic graphs constitute most of the molecular graphs. In this section, by considering some classes of these graphs, we will state and 
prove some bounds in terms of first and second exponential Zagreb, sum version of the first exponential Zagreb, and multiplicative exponential Zagreb indices. We remind that results on extremal multiplicative Zagreb indices for trees, unicyclic, and bicyclic graphs are presented, for instance, in $[18,19]$ and citations therein.

We also remind that an acyclic graph is a graph having no graph cycles, and they are actually bipartite as well as a connected acyclic graph is known as a tree. On the contrary, the unicyclic graph is a connected graph containing exactly one cycle. A connected unicyclic graph is, therefore, a pseudotree that is not a tree. We may refer [1] for the details of these terminology.

In the following, we will first see the results for some special classes of unicyclic graphs and then see the results for some special classes of acyclic graphs. Let $T\left(n, n_{1}, t\right)$ be a tree of order $n$ with the vertex set $V(G)=\left\{v_{1}, v_{2}, \ldots, v_{n}\right\}$ obtained from the path $v_{1} v_{2} \ldots v_{k+n_{1}}$ by appending the path $v_{k+n_{1}+1} \ldots v_{n}$ to vertex $v_{k}$ for $n_{1} \geq 1$ and $2 \leq k \leq n-n_{1}-1$. Thus, $U_{n}=T(n, n-3,2)$ is another class of tree graphs. So, these are some classes of acyclic graphs. After all, first and second additive and multiplicative exponential Zagreb indices of above acyclic graphs can be compared as follows.

Theorem 15. For the tree graphs $U_{n}$ and $T\left(n, n_{1}, k\right)$ with $n$-vertex, where $n_{1} \geq 1$ and $2 \leq k \leq n-n_{1}-1$, we have the following equalities and inequalities:

(i) $E M_{1}\left(U_{n}\right)=E M_{1}\left(T\left(n, n_{1}, k\right)\right)$ and $E M_{2}\left(U_{n}\right)<E M_{2}$ $\left(T\left(n, n_{1}, k\right)\right)$

(ii) $E M_{1}^{*}\left(U_{n}\right)<E M_{1}^{*}\left(T\left(n, n_{1}, k\right)\right)$

(iii) $E \Pi_{1}\left(U_{n}\right)=E \Pi_{1}\left(T\left(n, n_{1}, k\right)\right)$ and $E \Pi_{2}\left(U_{n}\right)<E \Pi_{2}$ $\left(T\left(n, n_{1}, k\right)\right)$

Proof. Let us consider $U_{n}$ and $T\left(n, n_{1}, k\right)$ as stated in the theorem.

(i) Let $n_{i}$ be the number of the vertices with $i$ degree. Then, all $n_{i}$ 's are equal to each other in $T\left(n, n_{1}, k\right)$ and $U_{n}$ which implies that $E M_{1}\left(U_{n}\right)=E M_{1}$ $\left(T\left(n, n_{1}, k\right)\right)$ by the equality in (1). Furthermore, to see the truthfulness of the second inequality, it is enough to apply the second part of (1) into the definitions of $U_{n}$ and $T\left(n, n_{1}, k\right)$. Therefore, we have $E M_{2}\left(U_{n}\right)=2 e^{2}+e^{3}+(n-6) e^{4}+2 e^{6}$ and $E M_{2}$ $\left(T\left(n, n_{1}, k\right)\right)=3 e^{2}+(n-8) e^{4}+3 e^{6}$. Consequently, $E M_{2}\left(U_{n}\right)<E M_{2}\left(T\left(n, n_{1}, k\right)\right)$, as required.

(ii) Applying (2) into the definitions of $U_{n}$ and $T\left(n, n_{1}, k\right)$, we get $E M_{1}^{*}\left(U_{n}\right)=2 e^{3}+(n-5) e^{4}+2 e^{5}$ and $E M_{1}^{*}\left(T\left(n, n_{1}, k\right)\right)=3 e^{3}+(n-8) e^{4}+3 e^{5}$ that clearly implies the inequality.

(iii) Similarly as in $i$, by considering the first part of (3), to verify first equality it is enough to see all $n_{i}$ 's are equal to each other in $T\left(n, n_{1}, k\right)$ and $U_{n}$ where $n_{i}$ is the number of the vertices with $i$ degree. Additionally, for the second inequality, it is sufficient to see that $E \Pi_{2}\left(U_{n}\right) / E \Pi_{2}\left(T\left(n, n_{1}, k\right)\right)=e^{2.2+1.3+2.6+4}$. $(n-6) / e^{2.3+3.6+4 .(n-8)}>1$.
Since a unicyclic graph has exactly one cycle, the number of vertices and edges are equal. Let $U_{n, k}$ be the set of all unicyclic graphs with $n$ vertices and girth $k$, where $n, k \geq 3$. Suppose also that $L_{n, k}$ and $H_{n, k}$ are the subsets of $U_{n, k}$. In here, we actually have $L_{n, k}=C_{k} \cup P_{n-k+1}$, and the unicyclic graph $H_{n, k}$ is obtained from $C_{k}$ by adding $(n-k)$ pendant vertices to a vertex of $C_{k}$.

We note that $L_{n, k}=C_{k}$ if $k=n$ and $H_{n, k}=L_{n, k}$ if $k=n-1$. So, we suppose that $3 \leq k \leq n-2$ in the following theorem.

Theorem 16. For graphs $L_{n, k}$ and $H_{n, k}$ with $n$ vertices and girth $k$ such that $n, k \geq 3$, we have

$$
E M_{1}\left(H_{n, k}\right) \geq E M_{1}\left(L_{n, k}\right) \text { and } E \Pi_{1}\left(H_{n, k}\right) \geq E \Pi_{1}\left(L_{n, k}\right) .
$$

Proof. Let $n_{i}$ be the number of the vertices with degree $i$. For the graphs $H_{n, k}$ and $L_{n, k}$, these numbers are $n_{1}=n-k$, $n_{2}=2 k-n$, and $n_{3}=n-k$ and $n_{1}=1, n_{2}=n-2$, and $n_{3}=1$, respectively. Consequently,

$$
\begin{aligned}
E M_{1}\left(H_{n, k}\right)-E M_{1}\left(L_{n, k}\right)= & (n-k) e^{1^{2}}+(2 k-n) e^{2^{2}} \\
& +(n-k) e^{3^{2}} \\
& -\left[1 . e^{1^{2}}+(n-2) e^{2^{2}}+1 . e^{3^{2}}\right] \\
\geq & e^{9}+4 e^{4}+e \geq 0,
\end{aligned}
$$

by the assumption $3 \leq k \leq n-2$.

To verify $E \Pi_{1}\left(H_{n, k}\right) \geq E \Pi_{1}\left(L_{n, k}\right)$, it is sufficient to see that

$$
\frac{E \Pi_{1}\left(L_{n, k}\right)}{E \Pi_{1}\left(H_{n, k}\right)}=\frac{e^{4 n+2}}{e^{6 n-2 k}}=e^{-2} \leq 1,
$$

since $k \leq n-2$. Hence, the result is obtained.

\section{Results on Graph Operations}

As mentioned in [30], some chemically interesting graphs can be obtained by different graph operations which are applied onto some general or particular graphs, so it is essential to study such graph operations to understand how they are related to the corresponding topological indices of the original graphs. So far, first and second Zagreb indices have been largely studied under some graph operations (see, for instance, $[3,8,11,30-35])$.

In this section, we will mainly compute the first and second multiplicative exponential Zagreb indices (see equation (3)) in specific graph extensions (or operations) such as Cartesian product $G \times H$, join $G+H$, lexicographic product $G[H]$, tensor product $G \otimes H$, symmetric difference $G \oplus H$, and finally the corona product $G * H$ for any graphs $G$ and $H$ (we refer any fundamental sources for the definitions of these operations). 
We have the following known results about these above operations which will be needed for our proofs.

Lemma 5 (see [36]). For any graphs $G$ and $H$, the following hold:
(i) $|V(G \times H)|=|V(G \oplus H)|=|V(G \otimes H)|=|V(G \quad[H])|$ $=|V(G)||V(H)|$
(ii) $|E(G \times H)|=|E(G)||V(H)|+|V(G)||E(H)|$
(iii) $|E(G+H)|=|E(G)|+|E(H)|+|V(G)||V(H)|$
(iv) $|E(G[H])|=|E(G)||V(H)|^{2}+|V(G)||E(H)|$
(v) $|E(G \oplus H)|=|E(G)||V(H)|^{2}+|V(G)|^{2}|E(H)|-4 \mid E$ $(G)|| E(H) \mid$

(vi) $G \times H$ is connected if and only if $G$ and $H$ are connected

Lemma 6 (see [37]). For any graphs $G$ and $H$, the following hold:

(1) If $(a, b) \in V(G \times H)$, then $\operatorname{deg}_{G \times H}(a, b)=\operatorname{deg}_{G}$ $(a)+\operatorname{deg}_{H}(b)$

(2) If $(a, b) \in V(G[H])$, then $\operatorname{deg}_{G[H]}(a, b)=|V(H)|$ $\operatorname{deg}_{G}(a)+\operatorname{deg}_{H}(b)$
(3) If $(a, b) \in V(G \oplus H)$, then

$$
\begin{aligned}
\operatorname{deg}_{G \oplus H}(a, b)= & |V(H)| \operatorname{deg}_{G}(a)+|V(G)| \operatorname{deg}_{H}(b) \\
& -2 \operatorname{deg}_{G}(a) \operatorname{deg}_{H}(b) .
\end{aligned}
$$

(4) If $a \in V(G+H)$, then $\operatorname{deg}_{G+H} \quad(a)=$ $\left\{\begin{array}{ll}\operatorname{deg}_{G}(a)+|V(H)| & \text { for } a \in V(G) \\ \operatorname{deg}_{H}(a)+|V(G)| & \text { for } a \in V(H)\end{array}\right.$.

Theorem 17. Let $G$ and $H$ be two graphs. Then, we have

$$
\begin{aligned}
& E \Pi_{1}(G \times H)=\left(\Pi_{1}(G)\right)^{|V(H)|}\left(\Pi_{1}(H)\right)^{|V(G)|} e^{8 E(G) E(H)} \text { and } \\
& E \Pi_{2}(G \times H)=e^{3\left(|E(H)| \cdot M_{1}(G)+|E(G)| M_{1}(H)\right)+M_{2}(H)|V(G)|+M_{2}(G)|V(H)|} .
\end{aligned}
$$

Proof. In the following proof, we will consider the facts in Lemma 5 (i) and (ii) and Lemma 6 (1).

Now, by adapting the first part of equation (3) in the Cartesian product, we obtain

$$
\begin{aligned}
E \Pi_{1}(G \times H) & =\prod_{u \in V(G \times H)} e^{d_{G \times H}^{2}(u)}=\prod_{(a, b) \in V(G \times H)} e^{d_{G \times H}^{2}((a, b))} \\
& =\prod_{a \in V(G) b \in V(H)} e^{\left(d_{G}(a)+d_{H}(b)\right)^{2}}=\prod_{a \in V(G) b \in V(H)} e^{d_{G}^{2}(a)+d_{H}^{2}(b)+2 d_{G}(a) d_{H}(b)} \\
& =\prod_{b \in V(H)} \prod_{a \in V(G)} e^{d_{G}^{2}(a)} \prod_{a \in V(G)} \prod_{b \in V(H)} e^{d_{H}^{2}(b)} \prod_{a \in V(G)} \prod_{b \in V(H)} e^{2 d_{G}(a) d_{H}(b)} \\
& =\prod_{b \in V(H)} \Pi_{1}(G) \prod_{a \in V(G)} \Pi_{1}(H) \prod_{a \in V(G)} e^{2 d_{G}(a) 2 E(H)} \\
& =\left(\prod_{1}(G)\right)^{|V(H)|}\left(\Pi_{1}(H)\right)^{|V(G)|} e^{8 E(G) E(H)} .
\end{aligned}
$$


Similarly, by adapting the second part of equation (3) in the Cartesian product, we obtain

$$
\begin{aligned}
E \Pi_{2}(G \times H)= & \prod_{u v \in E(G \times H)} e^{d_{G \times H}(u) d_{G \times H}(v)}=\prod_{(a, b)(c, d) \in E(G \times H)} e^{d_{G \times H}((a, b)) d_{G \times H}((c, d))} \\
= & \prod_{a \in V(G) b d \in E(H)} e^{d_{G \times H}((a, b)) d_{G \times H}((a, d))} \cdot \prod_{a c \in E(G) b \in V(H)} e^{d_{G \times H}((a, b)) d_{G \times H}((c, b))} \\
= & \prod_{a \in V(G)} \prod_{b d \in E(H)} e^{\left(d_{G}(a)+d_{H}(b)\right)\left(d_{G}(a)+d_{H}(d)\right)} \cdot \prod_{a c \in E(G)} \prod_{b \in V(H)} e^{\left(d_{G}(a)+d_{H}(b)\right)\left(d_{G}(c)+d_{H}(b)\right)} \\
= & \prod_{a \in V(G)}\left(e^{d_{G}^{2}(a)}\right)^{|E(H)|} \cdot \prod_{b d \in E(H)} e^{d_{G}(a)\left(d_{H}(b)+d_{H}(d)\right)} \cdot e^{d_{H}(b) d_{H}(d)} \\
& \cdot \prod_{b \in V(H)}\left(e^{d_{H}^{2}(b)}\right)^{|E(G)|} \prod_{a c \in E(G)} e^{d_{H}(b)\left(d_{G}(a)+d_{G}(c)\right)} \cdot e^{d_{G}(a) d_{G}(c)} \\
= & \prod_{a \in V(G)} e^{|E(H)| \cdot d_{G}^{2}(a)} \cdot e^{d_{G}(a) M_{1}(H)} \cdot e^{M_{2}(H)} \cdot \prod_{b \in V(H)} e^{|E(G)| d_{H}^{2}(b)} \cdot e^{d_{H}(b) M_{1}(G)} \cdot e^{M_{2}(G)} \\
= & e^{|E(H)| \cdot M_{1}(G)} \cdot e^{2|E(G)| M_{1}(H)} \cdot e^{M_{2}(H)|V(G)|} \cdot e^{|E(G)| M_{1}(H)} \cdot e^{2|E(H)| M_{1}(G)} \cdot e^{M_{2}(G)|V(H)|} \\
= & e^{3\left(|E(H)| \cdot M_{1}(G)+E(G) \mid M_{1}(H)\right)+M_{2}(H)|V(G)|+M_{2}(G)|V(H)|} \cdot
\end{aligned}
$$

Hence, the result is obtained.

Theorem 18. For any graphs $G$ and $H$, let us consider the join graph $G+H$ having vertex and edge sets $V(G+H)=$
$V(G) \cup V(H) \quad$ and $\quad E(G+H)=E(G) \cup E(H) \cup\{x y:$ $x \in V(G), y \in V(H)\}$, respectively. Thus, the first and second multiplicative exponential Zagreb indices are defined by

$$
\begin{aligned}
& E \Pi_{1}(G+H)=E \Pi_{1}(G) \cdot E \Pi_{1}(H) \cdot e^{|V(H)||V(G)|(|V(H)|+|V(G)|)+4(|V(H)||E(G)|+|V(G)||E(H)|)} \\
& E \Pi_{2}(G+H)=E \Pi_{2}(G) \cdot E \Pi_{2}(H) \cdot e^{|V(H)|^{2}(|V(G)|+|E(G)|)} \cdot e^{|V(G)| M_{2}(H)} \cdot e^{|V(H)| M_{2}(G)} \cdot . e^{|E(H)|\left(|V(G)|^{2}+4|E(G)|+2|V(H)|\right)} \cdot e^{2|V(G)||E(G)|} .
\end{aligned}
$$

Proof. With the help of Lemma 5 (i) and (iii) and Lemma 6 (4) and also adapting the first part of Equation (3) in join graphs, we have

$$
\begin{aligned}
E \Pi_{1}(G+H) & =\prod_{u \in V((G+H)} e^{d_{G+H}^{2}(u)}=\prod_{u \in V(G)} e^{d_{G+H}^{2}(u)} \cdot \prod_{u \in V(H)} e^{d_{G+H}^{2}(u)} \\
& =\prod_{u \in V(G)} e^{\left(d_{G}(u)+V(H) \mid\right)^{2}} \cdot \prod_{u \in V(H)} e^{\left(d_{H}(u)+|V(G)|\right)^{2}} \\
& =\prod_{u \in V(G)} e^{d_{G}^{2}(u)+2 d_{G}(u)|V(H)|+\left.V(H)\right|^{2}} \cdot \prod_{u \in V(H)} e^{d_{H}^{2}(u)+2 d_{H}(u)|V(G)|+\left.V(G)\right|^{2}} \\
& =E \prod_{1}(G) \cdot e^{|V(H)|^{2}|V(G)|} \cdot e^{4|V(H)||E(G)|} \cdot E \prod_{1}(H) \cdot e^{|V(G)|^{2}|V(H)|} \cdot e^{4|V(G)||E(H)|} \\
& =E \Pi_{1}(G) \cdot E \Pi_{1}(H) \cdot e^{|V(H)||V(G)|(|V(H)|+|V(G)|)+4(|V(H)||E(G)|+|V(G)||E(H)|)} .
\end{aligned}
$$


Again considering same lemmas and adapting the second part of Equation (3) in join graphs,

$$
\begin{aligned}
E \Pi_{2}(G+H)= & \prod_{u v \in E(G+H)} e^{d_{G+H}(u) d_{G+H}(v)} \\
= & \prod_{u v \in E(G)} e^{d_{G+H}(u) d_{G+H}(v)} \prod_{u v \in E(H)} e^{d_{G+H}(u) d_{G+H}(v)} \prod_{u \in V(G) v_{v \in V}(H)} e^{d_{G+H}(u) d_{G+H}(v)} \\
= & \prod_{u v \in E(G)} e^{\left(d_{G}(u)+|V(H)|\right)\left(d_{G}(v)+V(H) \mid\right)} \prod_{u v \in E(H)} e^{\left(d_{H}(u)+V(G) \mid\right)\left(d_{H}(v)+V(G) \mid\right)} \\
& \cdot \prod_{u \in V(G) v \in V(H)} e^{\left(d_{G}(u)+|V(H)|\right)\left(d_{H}(v)+V(G) \mid\right)} \\
= & E \prod_{2}(G) \cdot e^{|V(H)|^{2}|E(G)|} \cdot e^{|V(H)| M_{2}(G)} \cdot E \Pi_{2}(H) \cdot e^{|V(G)|^{2}|E(H)|} \cdot e^{|V(G)| M_{2}(H)} \\
& \cdot e^{(|V(G)||V(H)|)^{2}} \cdot e^{2(|V(G)||E(G)|+|V(H) \| E(H)|)} \cdot e^{4|E(G)||E(H)|} \cdot
\end{aligned}
$$

This completes the proof.

Theorem 19. Let $G$ and $H$ be graphs. Then, the following holds:

$$
\begin{aligned}
E \Pi_{1}(G[H])= & e^{|V(H)|\left(|V(H)|^{2} M_{1}(G)+8|E(H)||E(G)|\right)+V(G) \mid M_{1}(H)}, \\
E \Pi_{1}(G \oplus H)= & e^{|V(G)|^{3} M_{1}(H)} \cdot e^{|V(H)|^{3} M_{1}(G)} \cdot \prod_{u \in V(G) v \in V(H)} e^{2 d_{G}(u) d_{H}(v)\left(|V(G)|-2 d_{G}(u)\right)\left(|V(H)|-2 d_{H}(v)\right)}, \\
E \Pi_{1}(G \otimes H)= & e^{|V(G)|^{3} M_{1}(H)} \cdot e^{|V(H)|^{3} M_{1}(G)} \cdot e^{8|V(G)||V(H)||E(G)||E(H)|} \cdot e^{M_{1}(H) M_{1}(G)} \\
& \cdot e^{-4|V(H)||E(H)| M_{1}(G)} \cdot e^{-4|V(G)||E(G)| M_{1}(H)} .
\end{aligned}
$$

Proof. For simplicity, let us use the following labellings for only in this proof:

$$
\begin{gathered}
G[H] \longrightarrow \mu, \quad G \oplus H \longrightarrow \eta, \quad G \otimes H \longrightarrow \gamma, \\
|V(G)| \longrightarrow v_{1}, \quad|E(G)| \longrightarrow{ }_{1}, \quad M_{1}(G) \longrightarrow m_{G}, \\
|V(H)| \longrightarrow v_{2}, \quad|E(H)| \longrightarrow e_{2}, \quad M_{1}(H) \longrightarrow m_{H} .
\end{gathered}
$$

Firstly, let us consider the case $\mu$ by taking into account Lemma 5 (i) and (iv) and Lemma 6 (2). So,

$$
\begin{aligned}
E \Pi_{1}(\mu) & =\prod_{u \in V(\mu)} e^{d_{\mu}^{2}(\mu)}=\prod_{(a, b) \in V(\mu)} e^{d_{\mu}^{2}((a, b))}=\prod_{a \in V(G) b \in V(H)} e^{\left(v_{2} d_{G}(a)+d_{H}(b)\right)^{2}} \\
& =\prod_{a \in V(G) b \in V(H)} e^{v_{2}^{2} d_{G}^{2}(a)+d_{H}^{2}(b)+2 v_{2} d_{G}(a) d_{H}(b)} \\
& =\prod_{b \in V(H)} e^{v_{2}^{2} m_{G}} \cdot \prod_{a \in V(G)} e^{m_{H}} \cdot \prod_{a \in V(G)} e^{2 v_{2} 2 e_{1} d_{G}(a)}=e^{v_{2}^{2} v_{1} m_{G}} \cdot e^{v_{1} m_{H}} \cdot e^{2 v_{2} 2 e_{2} 2 e_{1}} \\
& =e^{v_{2}\left(v_{2}^{2} m_{G}+8 e_{2} e_{1}\right)+v_{1} m_{H}}=E \prod_{1}(G) \cdot e^{v_{2}^{2} v_{1}} \cdot e^{4 v_{2} e_{1}} \cdot E \Pi_{1}(H) \cdot e^{v_{1}^{2} v_{2}} \cdot e^{4 v_{1} e_{2}} \\
& =E \Pi_{1}(G) \cdot E \Pi_{1}(H) \cdot e^{v_{2} v_{1}\left(v_{2}+v_{1}\right)+4\left(v_{2} e_{1}+v_{1} e_{2}\right)} .
\end{aligned}
$$


Now, with the help of Lemma 5 (i) and (v) and Lemma 6 (3), let us show truthfulness of the second equality:

$$
\begin{aligned}
E \Pi_{1}(\eta) & =\prod_{(u, v) \in V(\eta)} e^{d_{\eta}^{2}((u, v))}=\prod_{u \in V(G) v \in V(H)} e^{\left(v_{1} d_{G}(v)+v_{2} d_{G}(u)-2 d_{G}(u) d_{H}(v)\right)^{2}} \\
& =\prod_{u \in V(G) \in V(H)} e^{v_{1}^{2} d_{H}^{2}(v)+v_{2}^{2} d_{G}^{2}(u)+2 v_{1} v_{2} d_{G}(u) d_{H}(v)+4 d_{G}^{2}(u) d_{H}^{2}(v)-4 d_{G}(u) d_{H}(v) v_{1} d_{H}(v)-4 d_{G}(u) d_{H}(v) v_{2} d_{G}(u)} \\
& =\prod_{u \in V(G)} e^{v_{1}^{2} m_{H}} \cdot \prod_{v \in V(H)} e^{v_{2}^{2} m_{G}} \cdot \prod_{u \in V(G) v \in V(H)} e^{2 d_{G}(u) d_{H}(v)\left[v_{1}-2 d_{G}(u)\right]\left[v_{2}-2 d_{H}(v)\right]} \\
& =e^{v_{1}^{3} m_{H}} \cdot e^{v_{2}^{3} m_{G}} \cdot \prod_{u \in V(G) v \in V(H)} e^{2 d_{G}(u) d_{H}(v)\left[v_{1}-2 d_{G}(u)\right]\left[v_{2}-2 d_{H}(v)\right]} .
\end{aligned}
$$

For the final step,

$$
E \Pi_{1}(\gamma)=\prod_{(u, v) \in V(\gamma)} e^{d_{\gamma}^{2}((u, v))}=\prod_{u \in V(G) v \in V(H)} e^{\left(v_{1} d_{G}(v)+v_{2} d_{G}(u)-d_{G}(u) d_{H}(v)\right)^{2}}=e^{v_{1}^{3} m_{H}} \cdot e^{v_{2}^{3} m_{G}} \cdot e^{8 v_{1} v_{2} e_{1} e_{2}} \cdot e^{m_{H} m_{G}} \cdot e^{-4 v_{2} e_{2} m_{G}} \cdot e^{-4 v_{1} e_{1} m_{H}}
$$

as required.

In the following final theorem, we are going to calculate the first and second multiplicative exponential Zagreb indices for the corona product.
Theorem 20. Let $G$ and $H$ be graphs, and let us consider their corona product; then, $G^{*} H$. In this case,

$$
\begin{aligned}
& E \Pi_{1}(G * H)=e^{|V(H)||V(G)|(|V(H)|+1)+M_{1}(G)+4(|V(H)||E(G)|+|V(G)||E(H)|)+M_{1}(H)|V(G)|} \\
& E \Pi_{2}(G * H)=e^{|V(H)|^{2}(|E(G)|+|V(G)|)+|V(G)|\left(|E(H)|+M_{1}(H)+M_{2}(H)\right)} \cdot e^{2|E(G)|(2|E(H)|+1)+M_{2}(G)+|V(H)|\left(M_{1}(G)+2|V(G)||E(H)|\right)} .
\end{aligned}
$$

Proof. Usually, we will apply Equation (3) into the definition of corona product in our calculations. For simplicity, let us label $G^{*} H$ by $\kappa$ :

$$
\begin{aligned}
E \Pi_{1}(\kappa) & =\prod_{u \in V(\kappa)} e^{d_{\kappa}^{2}(u)}=\prod_{u \in V(G)} e^{d_{\kappa}^{2}(u)}+\prod_{i=1}^{|V(G)|} \prod_{u \in V(H, i)} e^{d_{\kappa}^{2}(u)} \\
& =\prod_{u \in V(G)} e^{\left(d_{G}(u)+\mid V(H)\right)^{2}}+\prod_{i=1}^{|V(G)|} \prod_{u \in V(H, i)} e^{\left(d_{G}(u)+1\right)^{2}} \\
& =\prod_{u \in V(G)} e^{d_{G}^{2}(u)+|V(H)|^{2}+2|V(H)| d_{G}(u)}+\prod_{i=1}^{|V(G)|} \prod_{u \in(H, i)} e^{d_{G}^{2}(u)+1+2 d_{G}(u)} \\
& =e^{|V(H)|^{2}|V(G)|+M_{1}(G)+4|V(H)||E(G)|} \cdot e^{|V(H)||V(G)|+M_{1}(H)|V(G)|+4|V(G)||E(H)|} .
\end{aligned}
$$


Additionally,

$$
\begin{aligned}
E \Pi_{2}(\kappa) & =\prod_{u v \in E(\kappa)} e^{d_{\kappa}(u) d_{\kappa}(v)} \\
& =\prod_{u v \in E(G)} e^{d_{\kappa}(u) d_{\kappa}(v)} \cdot \prod_{i=1}^{|V(G)|} \prod_{u v \in E(H)} e^{d_{\kappa}(u) d_{\kappa}(v)} \cdot \prod_{u v \in \mathscr{P}} e^{d_{\kappa}(u) d_{\kappa}(v)},
\end{aligned}
$$

where $\mathscr{P}=\{u v: u \in V(G), v \in V(H, i)\}$ with the index $i=\{1,2, \ldots,|V(G)|\}$.

$$
\begin{aligned}
\prod_{u v \in E(G)} e^{d_{\kappa}(u) d_{\kappa}(v)} & =\prod_{u v \in E(G)} e^{\left(d_{G}(u)+V(H) \mid\right)\left(d_{G}(v)+|V(H)|\right)}=e^{|V(H)|^{2}|E(G)|+M_{2}(G)+V(H) \mid M_{1}(G)}, \\
\prod_{i=1}^{|V(G)|} \prod_{u v \in E(H)} e^{d_{\kappa}(u) d_{\kappa}(v)} & =\prod_{i=1}^{|V(G)|} \prod_{u v \in E(H)} e^{\left(d_{H}(u)+1\right)\left(d_{H}(v)+1\right)}=e^{|V(G)|\left(|E(H)|+M_{1}(H)+M_{2}(H)\right)}, \\
\prod_{u v \in \mathscr{P}} e^{d_{\kappa}(u) d_{\kappa}(v)} & =\prod_{i=1}^{|V(G)|} \prod_{u \in V(G)} e^{\left(d_{G}(u)+V(H)\right.}
\end{aligned}
$$

Equations (40)-(42) together give the required equality. Hence, the result is obtained.

\section{Conclusion and Future Problems}

By introducing new graph invariants (see Definition 1) under the name of "new generation exponential Zagreb indices," in here, we obtained some nice equality and inequality results in each section in terms of these new indices.
However, there are still some investigations need to be done for future studies. For example, by changing the graphs or graph operations used in this paper, the affection of these new indices can be checked. Additionally, the indices presented in Definition 1 may define another exponential Zagreb co-indices as in the following.

For separate vertices $u$ and $v$, the additive and multiplicative exponential Zagreb coindices are defined by

$$
\begin{aligned}
& \overline{E M_{1}}(G)=\sum_{u v \notin E(G)} e^{\operatorname{deg}(u)+\operatorname{deg}(v)}, \\
& \overline{E \Pi_{1}}(G)=\prod_{u v \notin E(G)} e^{\operatorname{deg}(u)+\operatorname{deg}(v)},
\end{aligned}
$$

Hence, all results presented and proved in this paper can also be checked with (43).

Also, creativity of chemical energy in terms of these new invariants can be determined. Finally, the application of these new indices can be controlled and optimized in QSPR/ QSAR studies.

\section{Data Availability}

No data were used to support this study.

\section{Conflicts of Interest}

The authors declare that they have no conflicts of interest.
$\left.\begin{array}{l}\overline{E M_{2}}(G)=\sum_{u v \notin E(G)} e^{\operatorname{deg}(u) \operatorname{deg}(v)}, \\ \overline{E \Pi_{2}}(G)=\prod_{u v \notin E(G)} e^{\operatorname{deg}(u) \operatorname{deg}(v)}\end{array}\right\}$

\section{References}

[1] F. Harary, Graph Theory, Reading, Addison-Wesley, Boston, MA, USA, 1994.

[2] J. Devillers and A. T. Balaban, Eds., Topological Indices and Related Descriptors in QSAR and QSPR, Gordon and Breach, Amsterdam, Netherlands, 1999.

[3] M. Bindushree, V. Lokesha, A. S. Cevik, and P. S. Ranjini, "Zagreb polynomial of three graph operators," Filomat, vol. 30, no. 7, pp. 1979-1986, 2016.

[4] I. Gutman and N. Trinajstić, "Graph theory and molecular orbitals, total $\pi$-electron energy of alternant hydrocarbons," Chemical Physics Letters, vol. 17, no. 4, pp. 535-538, 1972. 
[5] I. Gutman, B. Ruscic, N. Trinajstic, and C. F. Wilcox, "Graph theory and molecular orbitals, XII. Acyclic Polyenes," The Journal of Chemical Physics, vol. 62, no. 9, pp. 3399-3405, 1975.

[6] S. Nikolic, G. Kovacevic, A. Milicevic, and N. Trinajstic, "The Zagreb indices 30 years after," Croatica Chemica Acta, vol. 76, pp. 113-124, 2003.

[7] K. C. Das, I. Gutman, and B. Zhou, "New upper bounds on Zagreb indices," Journal of Mathematical Chemistry, vol. 46, no. 2, pp. 514-521, 2009.

[8] K. C. Das, A. Yurttas, M. Togan, A. S. Cevik, and I. N. Cangul, "The multiplicative Zagreb indices of graph operations," Journal of Inequalities and Applications, vol. 2013, Article ID 90, 2013.

[9] K. C. Das, N. Akgunes, M. Togan, A. Yurttas, I. N. Cangul, and A. S. Cevik, "On the first Zagreb index and multiplicative Zagreb coindices of graphs," Analele Stiintifice Ale Universitatii Ovidius Constanta, vol. XXIV, no. 1, pp. 153-176, 2016.

[10] G. H. Fath-Tabar, "Old and new Zagreb indices of graphs," MATCH Communications in Mathematical and in Computer Chemistry, vol. 65, pp. 79-84, 2011.

[11] M. H. Khalifeh, H. Yousefi-Azari, and A. R. Ashrafi, "The first and second Zagreb indices of some graph operations," Discrete Applied Mathematics, vol. 157, no. 4, pp. 804-811, 2009.

[12] B. Liu and Z. You, "A survey on comparing Zagreb indices," MATCH Communications in Mathematical and in Computer Chemistry, vol. 65, pp. 581-593, 2011.

[13] A. Yurttas, M. Togan, V. Lokesha, I. N. Cangul, and I. Gutman, "Inverse problems for Zagreb indices," Journal of Mathematical Chemistry, vol. 57, pp. 609-615, 2019.

[14] B. Zhou and I. Gutman, "Further properties of Zagreb indices," MATCH Communications in Mathematical and in Computer Chemistry, vol. 54, pp. 233-239, 2005.

[15] Z. Zhou, "Remarks on Zagreb indices," MATCH Communications in Mathematical and in Computer Chemistry, vol. 57, pp. 591-596, 2007.

[16] M. Eliasi, A. Iranmanesh, and I. Gutman, "Multiplicative versions of first Zagreb index," MATCH Communications in Mathematical and in Computer Chemistry, vol. 68, pp. 217230, 2012.

[17] R. Todeschini and V. Consonni, "New local vertex invariants and molecular descriptors based on functions of the vertex degrees," MATCH Communications in Mathematical and in Computer Chemistry, vol. 64, pp. 359-372, 2010.

[18] K. Xu and H. Hua, "A unified approach to extremal multiplicative Zagreb indices for trees, unicyclic and bicyclic graphs," MATCH Communications in Mathematical and in Computer Chemistry, vol. 68, pp. 241-256, 2012.

[19] K. Xu and K. C. Das, "Trees, unicyclic and bicyclic graphs extremal with respect to multiplicative sum Zagreb index," MATCH Communications in Mathematical and in Computer Chemistry, vol. 68, pp. 257-272, 2012.

[20] K. C. Das, I. Gutman, and B. Horoldagva, "Comparing Zagreb indices and coindices of trees," MATCH Communications in Mathematical and in Computer Chemistry, vol. 68, pp. 189-198, 2012.

[21] A. R. Ashrafi, T. Doslic, and A. Hamzeh, "Extremal graphs with respect to the Zagreb coindices," MATCH Communications in Mathematical and in Computer Chemistry, vol. 65, pp. 85-92, 2011.

[22] H. Hua, A. R. Ashrafi, and L. Zhang, "More on Zagreb coindices of graphs," Filomat, vol. 26, pp. 1210-1220, 2012.
[23] Z. K. Vukicevic and G. Popivoda, "Chemical trees with extreme values of Zagreb indices and coindices," Iranian Journal of Mathematical Chemistry, vol. 5, no. 1, pp. 19-29, 2014.

[24] J. Rada, "Exponential vertex-degree-based topological indices and discrimination," MATCH Communications in Mathematical and in Computer Chemistry, vol. 82, pp. 29-41, 2019.

[25] R. Cruz and J. Rada, "The path and the star as extremal values of vertex-degree-based topological indices among trees," MATCH Communications in Mathematical and in Computer Chemistry, vol. 82, pp. 715-732, 2019.

[26] M. Zeng and H. Deng, The Maximal Tree with Respect to the Exponential of the Second Zagreb Index, https://arxiv.org/abs/ 2006.08892v1, 2020.

[27] K. Xu, K. C. Das, and K. Tang, "On the multiplicative Zagreb coindex of graphs," Opuscula Mathematica, vol. 33, no. 1, pp. 191-204, 2013.

[28] I. Gutman and K. C. Das, "The first Zagreb index after 30 years," MATCH Communications in Mathematical and in Computer Chemistry, vol. 50, pp. 83-92, 2004.

[29] K. C. Das and I. Gutman, "Some properties of the second Zagreb index," MATCH Communications in Mathematical and in Computer Chemistry, vol. 52, no. 1, pp. 103-112, 2004.

[30] V. Lokesha, S. Jain, A. S. Cevik, and I. N. Cangul, "New results on the F-index of graphs based on corona-type products of graphs," Proceedings of the Jangjeon Mathematical Society, vol. 23, no. 2, pp. 141-147, 2020.

[31] N. Akgunes and B. Cagan, "On the dot product of graphs over monogenic semigroups," Applied Mathematics and Computation, vol. 322, pp. 1-5, 2018.

[32] M. Azari and A. Iranmanesh, "Some inequalities for the multiplicative sum Zagreb index of graph operations," Journal of Mathematical Inequalities, vol. 9, no. 3, pp. 727-738, 2015.

[33] B. Basavanagoud and S. Patil, "A note on hyper-Zagreb index of graph operations," Iranian Journal of Mathematical Chemistry, vol. 7, no. 1, pp. 89-92, 2016.

[34] R. Frucht and F. Harary, "On the corona of two graphs," Aequationes Mathematicae, vol. 4, no. 3, pp. 322-325, 1970.

[35] G. H. Shirdel, H. Rezapour, and A. M. Sayadi, "The hyperZagreb index of graph operations," Iranian Journal of Mathematical Chemistry, vol. 4, no. 2, pp. 213-220, 2013.

[36] W. Imrich and S. Klavzar, Product Graphs: Structure and Recognition, John Wiley \& Sons, New York, NY, USA, 2000.

[37] M. H. Khalifeh, H. Yousefi-Azari, and A. R. Ashrafi, "The hyper-Wiener index of graph operations," Computers \& Mathematics with Applications, vol. 56, no. 5, pp. 1402-1407, 2008. 